\title{
COMPETENCIAS BÁSICAS Y TRANSVERSALES, NUEVAS DEMANDAS DE FORMACIÓN EN LA EDUCACIÓN SUPERIOR
}

Mtra. Irma Piña Jiménez:

"Prof. Titular "A" Drvisión de Estudios de Posgrado ENEO-UNAM

El presente articulo parte del cuestionamiento que se hace a los fines de la educación superior en el nuevo siglo, en concordancia con la presencia de la noción de competencia y las implicaciones que ésta tiene en la práctica educativa que se ocupa de la formación de los profesionales. Para ello retoma de manera especial, algunas aportaciones que el autor Bernard Rey de la Universidad Libre de Bruselas elabara, sobre las competencias transversales.

Posteriormente analiza algunos argumentos en torno a las preguntas icómo se propicia la formación del profesional competente? y Lcómo se adquieren las competencias? centrándose en el caso de la enfermería universitaria.

En el último apartado, se hace una reflexión respecto a la naturaleza de los contenidos no escritos que derivan del saber práctico profesional, cuya enseñanza posee la ventaja de no desvincularse de su práctica.

PALABRAS CLAVE: competencias, formación basada en competencias, aprendizaje in situ.

\section{Abstract}

This article arises from the questions made about higher education goals in the new century, according to the concept of competence and implications thereof in the educational practice focus on professional training. Some contributions of Bernard Rey, an author from the Free University of Brussels, set forth about transversals competencies, have been specially taken into consideration.

Afterwards, some arguments about how the training of a competent professional can be favored, and how said competencies can be acquired, have been reviewed, mainly for university nursing.

In the last part, a reflection about the nature of the no writing know-how coming from the professional practical knowledge is made, which teaching has the advantage of being inseparable of the practice.

KEY WORDS: competencies, competency bosed education, in situ learning. 


\section{INTRODUCCIÓN}

El tema de las competencias, abre nuevamente a la discusión de una antigua pregunta: ¿para qué educar? intentar dar respuesta a esta interrogante, requiere necesariamente ubicarse en las caracteristicas que presenta el contexto económico, político y social en la actualidad, como resultado del proceso de globalización, en el que las circunstancias externas del mundo impactan de alguna forma las condiciones locales y en el que la educación se vislumbra, como el recurso a través del cuál, los individuos del mundo podrán adquirir una gama de conocimientos clave, que les permitirán continuar vigentes en un mundo de permanente cambio y complejidad.

Los estudios de PISA (Programme for International Student Assesment por sus siglas en inglés) desarrollan una serie de indicadores, que se han propuesto determinar ien qué medida los individuos de 15 años, que viven en los diferentes continentes del mundo y que participan en diversos sistemas educativos, cuentan con los conocimientos fundamentales, que les permitirán desempeñar un papel constructivo en la sociedad, demostrando si son capaces de aplicarlos a las situaciones cotidianas de la vida! .

Esta medida, demanda al sistema educativo de cada país, que el trabajo educativo de los jóvenes, se traduzca en una formación que aporte las herramientas necesarias, que les permitan interactuar con el medio, con un bagaje cultural que trascienda el espacio áuliço e invada la vida diaria.

Se trata pues, de abandonar aquellas prácticas educativas que se limitan a "cubrir el programa" y que exponen a los alumnos a situaciones ficticias de aprendizaje, que no parecen estar garantizando, la traslación del modus operanti a las situaciones reales que lo demandan. En esta lógica Bernard Rey, apunta a las competencias transversales: veamos a qué se refiere esta noción.

\section{LA NOCIÓN DE COMPETENCIA Y EL REPLANTEA- MIENTO DE LOS FINES DE LA EDUCACIÓN.}

Bernard Rey² al referirse al tema de las competencias transversales, menciona que en ellas, si bien las diversas disciplinas están presentes, éstas constituyen sólo un medio para alcanzar un fin, mas no suponen la firralidad misma del conocimiento. Es decir, en las competencias transversales, confluyen diversas disciplinas que no son importantes per se, sino en la medida en que contribuyen a conformar aquellas competencias que el individuo ha de emplear en su vida personal, profesional o social.
Así, cada disciplina aporta elementos que le son propios, pero paralelamente se requiere trabajar en aquellas competencias que la disciplina no puede dar. Asl por ejemplo, un alumno puede recurrir al estudio de la anatomia y la fisiología, para preparar la exposición de un tema, pero requiere ejercitar paralelamente la competencia de la comunicación, si pretende que este tema sea comprendido por un auditorio, en su acepción visual y auditiva, así como configurar una estructura lógica, que facilite su comprensión y organización de la mejor forma.

El concepto sobre competencia que refiere el autor, señala que ". es la excelencia que se reconoce en el otro y que puede ser objeto de admiración":3 esta definición indica que si bien la competencia es accesible a la apreciación externa, se constituye desde la subjetividad. Por ello, su evidencia, no la reduce a una serie de conductas especificas y observables, que pueden descomponerse en movimientos elementales, como supone una noción de competencia centrada en la conducta observable.

"... Una competencia se define como un sistema de conocimientos conceptuales y procedurales, organizados como esquemas operacionales que permiten, frente a una familia de situaciones, la identificación de una tarea problema y su resolución mediante una acción eficaz... Lo que abre camino al concepto de un sujeto provisto de un equipo cognitivo complejo, que no se limita a la sedimentación de reacciones condicionadas".4.

Las competencias poseen un carácter generativo -indica el autor refiriendo a Noam Chomsky, - y poseen una potencialidad invisible, interior y personal, susceptible de generar una infinidad de actuaciones. Es decir, una persona competente posee una gama altamente estructurada de conocimientos en su interior, que le da la posibilidad de enfrentar un repertorio muy variado de situaciones problema, saliendo venturoso y que si bien en su actuar es exacto, pertinente y adecuado, dificilmente podría este sujeto expresar cómo logró llegar a realizar las cosas con tal sencillez y pericia, si se lo preguntáramos. Otro aspecto que el autor destaca de la noción de competencia, es que está ligada a un contenido y a una situación concretar y que lo que se espera es que el alumno, pueda transferirla a situaciones inéditas, es decir no enfrentadas con anterioridad. En este ültimo sentido Rey coincide con Phillipe Perrenoud, quien refiere la movilización de los conocimientos, como una de las aportaciones centrales del concepto de competencia, pese a que el autor afirma que se desconoce la forma a través de la cual lo haces. 
Esto supone dentro del contexto de la enseñanza y del aprendizaje, la necesidad de contar con espacios que muestren a los alumnos diversas situaciones sobre las que deban intervenir, ejercitando habilidades vinculadas con su hacer profesional. La posibilidad de generar un aprendizaje in situ, en el que el alumno se enfrenta a un problema real que lo invita a analizar el caso particular, y a determinar la manera en que deberá desplegar determinadas habilidades a partir de los conocimientos que posee.

Esto es, el alumno tiene que aprender a leer la realidad, y esto en si constituye una habilidad que traspasa los limites disciplinarios, aprendiendo a situarse ante un problema determinado, empleando los muchos o pocos elementos de que dispone. De ahi que el problema dependerá de la manera en que visualiza e identifica el problema, del sentido que el alumno le da y de la perspectiva desde la cuál se posiciona ante el problema, así como el o los aspectos sobre los que considera que puede intervenir, de manera que el alumno utiliza y resignifica los conocimientos y los saberes previamente adquiridos.

\section{SOBRE LA ADQUISICIÓN DE LAS COMPETENCIAS QUE REQUIERE EL. PROFESIONAL COMPETENTE.}

Considerando los aspectos anteriores, formar profesionales competentes reclama entre otras cosas a las instituciones de educación superior: la delimitación de las funciones y roles profesionales que requieren ser ponderados; delimitar los campos de conocimiento disciplinarios que sientan las bases en la generación de competencias transversales y ubicar en un nivel metodológico e instrumental, los problemas a situaciones que propiciarán el desencadenamiento de las competencias básicas que requieren poner en juego los profesionales.

Roe refiere la necesidad de combinar el modelo de input y de output que se centra en dos distintos ejes, el primero. en las disciplinas y el segundo en la práctica. División y separación que tradicionalmente se observa en las instituciones escolares y que como mencionamos anteriormente, no posibilita la movilización del conocimiento a las situaciones reales.

Este último aspecto, requiere la presencia de docentes con habilidades profesionales, que sean capaces de guiar al alumno en una serie de trabajos prácticos (demostraciones, trabajos de investigación, proyectos, ejercicios, etc.) y en prácticas supervisadas en escenarios reales de intervención profesional, como es el caso de la Enfermería. Roe refiere la necesidad de plantear un entrenamiento pro- fesional inicial, en el que el alumno es acompañado por un profesor; durante su práctica, realizando tareas, responsabilidades y ejercitando roles que requiere como profesional, pero sin asumir toda la responsabilidad que esto conlleva para con los pacientes. Se trata de un entrenamiento acompañado y supervisado por un tutor profesional.

Roe, si bien refiere sus aportaciones al caso del psicólogo competente, éstas bien pueden servir para el caso de la formación de profesionales de Enfermeria.".....El entrenamiento profesional inicial debe ofrecer a los que poseen las competencias básicas la oportunidad de comprometerse en el trabajo profesional, bajo la supervisión de psicólogos cualificados. La supervisión significa que un psicólogo cualificado asume la responsabilidad completa de vis a vis del aprendiz con el cliente. Pero la supervisión también sirve para promover el proceso de aprendizaje ofreciendo un modela de rol, entrenando al aprendiz durante la socialización y la ejecución en el rol laboral, dando feedback y estimulando la reflexión..."

De esta forma, podriamos afirmar que el conocimienta que se aporta a través de la práctica supervisada y del entrenamiento profesional inicial, constituye en sí un contenido y que éste puede ser enriquecido cualitativamente con los conocimientos disciplinares de la profesión, que aportan elementos para la construcción de las competencias transversales.

Paralelamente, en la mayoria de los casos la vinculación teoría práctica, no se hace presente en forma causal, en tanto los problemas que enfrenta el alumno aprendiz, son complejos y multireferenciados, debiendo recurrir a varias disciplinas para tratar de comprenderlos y descifrarlos. Asi por ejemplo, tendrá que atender un paciente adolescente, que presenta un cuadro patológico determinado, para lo cual sus conocimientos sobre patología, anatomía, fisiología y psicologia le proveen inicialmente de un marco referencial, que deberá aunarse al de una situación socio -cultural y económica determinada, que tiende a agravarlo (por ejemplo desnutrición, baja escolaridad, carencia de seguridad social, entomo familiar disfuncional, etc.) aspectos todos ellos, con los que el alumno deberá contender, tanto en su diagnóstico de cuidado como en su tratamiento.

\section{SOBRE LOS CONTENIDOS QUE APORTA LA EXPERIENCIA DIRECTA EN LA PRÁCTICA Y SU FORMULACIÓN NO ESCRITA.}

Las carreras con carácter profesionalizante como es el caso de la Enfermería, encuentran en la práctica toda una gama de conocimientos que no necesariamente se asien- 
tan en una determinada disciplina, se trata de un conjunto de saberes y habilidades que no necesariamente han sido escritos o documentados de manera: formal en un campo de conocimientos, de ahi que estos contenidos, no puedan ser repensados, analizados y discutidos colectivamente. "...La escritura de las cosas genera la posibilidad de analizarlas, discutirlas colectivamente, es una herramienta potente para apropiarse intelectualmente del mundo, en la medida en que ella fija los conceptos"t?

Así, una gama de saberes que son producto de la práctica, al no estar formalizados de manera escrita, sólo pueden ser enseñados a través del ejemplo y de la demostración de quien los domina, es decir, se entabla una relación educativa en el que se muestra el conocimiento al alumno, sin que medie un documento escrito sobre dicho contenido. En este sentido Bernard Rey afirma que la ausencia de la escritura, tiene un efecto en el aprendizaje, en tanto que el momento de enseñanza no se encuentra desvinculado de la práctica. Cuando está presente la escritura, aquello que se enseña, es revisado, valorado y estructurado en función de los sujetos de aprendizaje, es decir se habla de una transmisión con una estructura didáctica. Así, al objetivarse el saber se hace independiente de los ejecutantes, la escritura aisla los saberes de las prácticas.

John Dewey afirmaba desde principios del siglo XX, que la experiencia implica el principio de una actividad y simultáneamente el reconocimiento de las consecuencias que de. ella se derivan. "Este tipo de experiencia debe conducir.. al desarrollo natural e integrado de los conocimientos, del saber hacer y de la reflexión." De ahi que Jerome Bruner ${ }^{8}$ considere la experiencia directa y la mediatizada, como fuentes que determinan formas de conocimiento y habilidades, acordes a la forma en que son adquiridos. Es decir, el sujeta en su relación con el objeto de conocimiento construye representaciones del mismo y éstas representaciones a su vez derivan de la forma en que ambos interactúan, resultando determinados contenidos, habilidades que se interiorizan y esquemas de acción.

En este sentido el aprendizaje de contenidos captados por la vía de la experiencia directa, no puede ser sustituido por otras formas educativas, y si estamos interesados en formar profesionales competentes, las actividades vinculadas con la práctica deben estar presentes en diversas formas y escenarios del desarrollo curricular.

\section{CONSIDERACIONES FINALES}

El temas de las competencias, nos reubica en la importancia que tiene para la educación del nuevo siglo, formar para interactuar e intervenir en la vida cotidiana, sea desde el punto de vista profesional, sea como ciudadanos del mundo.

Siendo así, la formación de las enfermeras universitarias por su naturaleza como profesión eminentemente prácti$c a$, tiene amplias posibilidades de avanzar en este sentido, pues su enseñanza en los espacios reales de ejercicio profesional, es una actividad común que se encuentra desde el origen mismo de la profesión, en la que los entornos clínicos y comunitarios han estado presentes como espacios de aprendizaje in situ en los que los alumnos se enfrentan a situaciones reales, generadoras de habilidades y de las competencias básicas que requiere este profesional. Lo que sí resultará complejo es transitar a la formación de competencias transversales, pues esto reclama para los espacios educativos y de formación, una visión desde los actores del proceso educativo que implica desenmarcarse de los contextos particularizados que suponen las disciplinas especificas en las que tradicionalmente han sido formados los docentes considerar que la Enfermería como disciplina se constituyó en forma tardía, por lo que aún en el presente, se encuentra en debate este aspecto, en el que delimita su objeto de estudio y su método de trabajo, lo que ha contribuido en gran medida a darle a la Enfermería una nueva identidad.

\section{REFERENCIAS BIBLIOGRÁFICAS}

1 Organización para la cooperación y el desarrollo económico. Marcos teóricos de PISA. 2003: La medida de los conocimientos y destrezas en matemáticas, lectura, ciencias y resolución de problemaș. Ministerio de Educación y Ciencias. instituto Nacional de Evaluación y Calidad del Sistema Educativo, Madrid, 2004. 140pp.

2. Rey, Bernard. Las competencias transversales en cuestión. Trad. por Alejandro Madrid Zan. Escuela de Filosofía de la Universidad de Arcis, Chile, 2001. p.41

3 Íbidem, p. 17

4 Ibidem, p. 27

5 Perrenoud, Phillipe. Construir competencias desde la escuela Océano/ Dolmen Pedagogía, Caracas, 2002. p.35

6 Roe, Robert "¿Qué hace competente al psicólogo?", en Revista del Colegio Oficial de Psicólogos. The Netherlands, University of Nijmejen, Madrid, diciembre, No. 86, 2003. p.11

7 Rey, Bernard, Op. Cit. p. 15

8 Bruner, Jerome 5. y David R. OLSON. Aprendizaje por experiencia directa y aprendizaje por experiencia mediatizada, en Revista Perspectivas. UNESCO, Madrid, 1973. p.1

\section{DIRECCIÓN PARA CORRESPONDENCIA}

Irma Piña Jiménez: ipina@starmedia.com 